\title{
Multiphase CT Angiography: A Useful Technique in Acute Stroke Imaging-Collaterals and Beyond
}

\author{
(D) S. Dundamadappa, (D) K. lyer, (D) A. Agrawal, and (DD.J. Choi
}

\section{ABSTRACT}

SUMMARY: Multiphase CTA offers several important advantages over the traditional single-phase CTA technique in acute ischemic stroke, including improved detection of large-vessel occlusion, improved characterization of collateral status, improved tolerance of patient motion and poor hemodynamics, and higher interrater reliability. These benefits are gleaned at little additional cost in terms of time, risk to the patient, and capital expense. Existing data suggest that there are important benefits to using multiphase CTA in lieu of single-phase CTA in the initial vessel assessment of patients with acute stroke.

ABBREVIATIONS: AIS = acute ischemic stroke; $\mathrm{mCTA}=$ multiphase CTA; $\mathrm{sCTA}=$ single-phase CTA

$\mathrm{T}$ he introduction of IV reperfusion therapy for acute ischemic stroke (AIS) more than 20 years ago spawned an era of dramatic advances in the treatment of patients with AIS. Neuroimaging has consistently played a critical role in triaging patients with acute stroke by helping providers weigh the risks and benefits of treatment options. Important imaging findings during triage of patients with stroke have included intracranial hemorrhage, large-vessel occlusion, core infarct, penumbra, and collateral circulation status. Other less commonly used imaging findings such as thrombus length and thrombus perviousness have also assisted stroke treatment teams.

Neuroradiologists involved in the care of patients with stroke must be conversant with the diagnostic strengths and limitations of various stroke imaging modalities. The value of any imaging test should be considered in the context of its availability, acquisition and postprocessing speed, tolerance of suboptimal patient factors such as motion, and interrater reliability.

Multiple clinical trials have prompted the stroke community to recommend specific imaging modalities for various stroke scenarios, as stated in the latest 2019 American Heart Association/ American Stroke Association Guidelines for Management of AIS. ${ }^{1}$ In patients with AIS presenting within 6 hours of symptom onset and with a small core infarct on NCCT, CTA or MRA is advised to guide patient selection for mechanical thrombectomy

Received June 22, 2020; accepted after revision August 21.

From the Department of Radiology, University of Massachusetts Medical Center, Worcester, Massachusetts.

Please address correspondence to David Choi, MD, PhD, Department of Radiology, Room S2-824, University of Massachusetts Medical Center, 55 Lake Ave North,

Worcester MA 01655; e-mail: David.Choi@umassmemorial.org

- Indicates open access to non-subscribers at www.ajnr.org

http://dx.doi.org/10.3174/ajnr.A6889 without the need for additional modalities such as CTP. In patients presenting in the extended 6- to 24-hour window after onset and who have a large-vessel occlusion in the anterior circulation, CTP or diffusion-weighted MR imaging with or without MR perfusion is advised, as supported by the more recent DWI or CTP Assessment with Clinical Mismatch in the Triage of Wake-Up and Late Presenting Strokes Undergoing Neurointervention with Trevo (DAWN) and Endovascular Therapy Following Imaging Evaluation for Ischemic Stroke 3 (DEFUSE 3) trials. ${ }^{2,3}$ The Endovascular Treatment for Small Core and Proximal Occlusion Ischemic Stroke (ESCAPE) trial $^{4}$ and secondary analysis from the Multicenter Randomized Clinical Trial of Endovascular Treatment for Acute Ischemic Stroke in the Netherlands (MR CLEAN) trial ${ }^{5}$ have suggested that collateral status might also be considered when selecting patients for thrombectomy.

In patients with AIS, CTA has been the study of choice for the initial diagnosis of large-vessel occlusion and other important vascular pathology such as dissection. Multiphase CTA (mCTA) is a more recently studied, time-resolved CTA variant in which an arterial and 2 venous phases are acquired. mCTA offers several advantages over the traditional single-phase CTA technique (sCTA), including improved detection of large-vessel occlusion, ${ }^{6}$ higher interrater reliability, and improved characterization of collateral status. ${ }^{7}$ There has been increasing use of mCTA in recent clinical trials. ${ }^{4}$ Our institution, a comprehensive stroke center, adopted mCTA as its standard noninvasive vessel imaging technique for AIS several years ago.

In this review, we discuss the acquisition technique and interpretation of mCTA, its role in AIS patient assessment, its advantages over sCTA, and its limitations. We hope this review will help stroke providers understand the advantages of this evolving technique. 


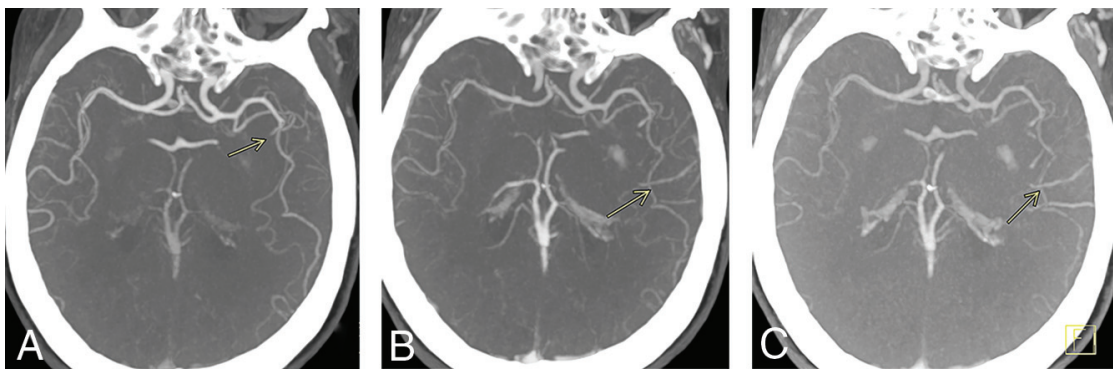

FIG 1. Delayed vessel sign. Peak arterial $(A)$, early venous $(B)$, and late venous $(C)$ phase axial MIP mCTA images show occlusion of a proximal M2 segment (arrow in A) of the left MCA. The delayed vessel sign is demonstrated in the early venous and late venous phases (arrows in $B$ and C), assisting in detection of occlusion.

\section{METHODS}

\section{Imaging Protocol for Acute Stroke}

At our institution, initial imaging for patients with AIS includes NCCT and mCTA with the goal of door-to-CT table time of less than 20 minutes. For patients presenting between 6 to 24 hours after onset of symptoms or when the time of onset is unknown, CTP is also performed. For a subset of patients (eg, those with strong contraindication to iodinated contrast or those with unclear correlation between imaging findings and neurologic signs), an abbreviated hyperacute MR imaging protocol may be performed instead (consisting of DWI, axial T2 FLAIR, and axial SWI with or without MRA).

Our mCTA protocol (Edge CT scanner; Siemens) is as follows:

- Contrast bolus of $80 \mathrm{cc}$ (Omnipaque 300), at the rate of $4 \mathrm{~mL} /$ $\mathrm{s}$ followed by a saline chaser.

- First phase (peak arterial phase): Coverage extends from the aortic arch through the vertex. Image acquisition is triggered by bolus monitoring with ROI in proximal descending aorta with a trigger value of $115 \mathrm{HU}$.

- Second phase (equilibrium/peak venous phase): Coverage extends from the skull base through the vertex performed 4 seconds after completion of the peak arterial phase scan.

- Third phase (late venous phase): Coverage extends from the skull base through the vertex performed 4 seconds after completion of the second phase.

mCTA imposes little burden at postprocessing, requiring only the creation of multiplanar MIP images of the 3 phases. Such MIP images can be generated automatically within minutes using standard vendor software. In the neck, coronal and sagittal plane MIP images are created with a thickness of $5 \mathrm{~mm}$ and an interslice gap of $2 \mathrm{~mm}$. In the head, axial plane MIP images are created with a thickness of $20 \mathrm{~mm}$ and an interslice gap of $3 \mathrm{~mm}$; MIP images in the coronal and sagittal planes are created with a thickness of $10 \mathrm{~mm}$ and an interslice gap of $3 \mathrm{~mm}$. On the PACS, head MIP images are best displayed for each plane with the 3 phases side by side. This arrangement facilitates appreciation of the dynamic behavior of vessel opacification over time. Collateral status is often best assessed on axial head MIP images.

Compared with sCTA, the additional time cost to acquire mCTA includes the scan time for the additional 2 phases of head CTA
( 3 seconds each), the time between phases ( 4 seconds each), and the time required for reconstruction of the additional head MIPs (2-3 minutes). At our institution, iterative reconstruction is turned off at mCTA to shorten the reconstruction time.

Vendor software is available that can create color-coded summation maps of mCTA MIP images, such as the Faststroke package Colorviz view (GE Healthcare). This tool combines information from all 3 phases by color coding vascular segments according to their peak phase of enhancement. These color-coded mCTA summation maps may facilitate assessment of perfusion delay, distal and multivessel occlusion, critical stenosis, and collateral flow patterns, particularly for less experienced readers. ${ }^{8}$ Although promising, such a tool is not required to realize the benefits of mCTA described in this review, and we have not yet adopted such a tool at our institution.

\section{DISCUSSION}

mCTA can be performed on most standard CT platforms. The technique is readily available, rapidly performed, needs no additional contrast material, requires no additional vendor package for specialized postprocessing (as is needed for CTP), and is relatively easy to interpret. mCTA is more accurate than sCTA in assessing collateral status, a useful factor for revascularization decisions in some patients with AIS. In addition, mCTA increases sensitivity for arteriovenous shunting and venous abnormalities.

\section{Sensitivity for Vessel Occlusion}

mCTA increases interrater agreement for both proximal (M1, A1 segment) and distal (M2, M3, A2 segment) vessel occlusions compared with sCTA, particularly for trainees, general radiologists, and neurologists. ${ }^{9,10} \mathrm{mCTA}$ improves the detection rate of distal vessel occlusions compared with sCTA by virtue of the "delayed vessel sign"10 (Figs 1 and 2). This sign refers to the delayed enhancement of a vessel segment distal to the point of an occlusion or critical stenosis. The "delayed vessel" reaches peak enhancement at a later phase than equivalent nonoccluded vessels, which can increase the conspicuity of distal vessel occlusions. Such occlusions are sometimes difficult to detect by sCTA regardless of reader experience. It is likely that this "delayed vessel" enhancement reflects retrograde flow via pial collaterals. The delayed vessel sign is less sensitive when there are poor collaterals in the ischemic territory, in occlusions of end arteries or perforator arteries, and with small cortical infarcts. ${ }^{10}$

Endovascular intervention has become more common place for some distal vessel occlusions, such as the M2 segment. The safety profile and clinical benefits for treating some M2 occlusions approach that of M1 occlusions. ${ }^{11-13}$ Occlusions of the M2 segment highlight the value of the improved distal vessel detection rate by mCTA compared with sCTA. Even in patients who are not candidates for revascularization therapy, the detection of a distal 
segment occlusion may provide added value to patient management, such as when there is unclear correlation between neurologic signs and imaging findings or when there is no core infarct at imaging.

Studies of the time needed for neuroradiologists, radiology trainees, and neurologists to diagnose a large-vessel occlusion by mCTA versus sCTA have favored mCTA. ${ }^{9,10}$ After a training period, readers on average consistently reported greater diagnostic confidence with and expended less time diagnosing large-vessel occlusion by mCTA compared with sCTA examinations. In a study of 2 radiology residents and 2 neuroradiologists, Byrne et $\mathrm{al}^{10}$ reported the average time needed for the radiology trainees to diagnose proximal M1 occlusions as 34 seconds by mCTA versus 72 seconds by sCTA. The average time for the radiology trainees to diagnose distal vessel occlusions (A2, M2, A3, M3) was 44 seconds by mCTA versus 99 seconds by sCTA. For the 2 neuroradiologists, the average time to diagnose proximal M1 occlusions was 29 seconds by mCTA versus 67 seconds by sCTA. Although the sheer number of additional source and MIP images generated by mCTA may seem discouraging at first, Byrne et $\mathrm{al}^{10}$ suggested that the diagnostic efficiency of mCTA may be related to the value of the delayed vessel sign.

\section{Length of Occlusion}

Accurate assessment of thrombus length has prognostic significance and assists in the planning and execution of thrombectomy. Thrombus burden is a predictor for infarct size, hemorrhagic transformation, and clinical outcome ${ }^{14}$ and has implications for therapy. Recanalization is unlikely for IV tPA when the thrombus
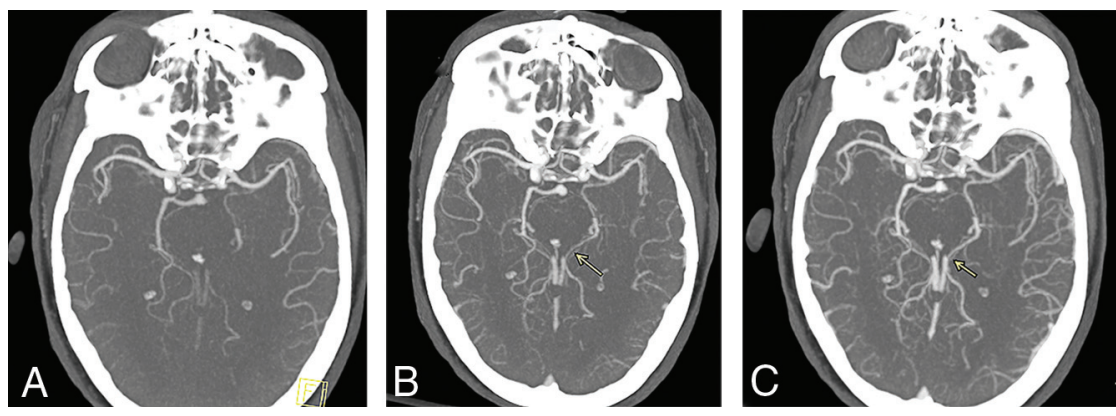

FIG 2. Axial arterial phase mCTA MIP image of a left PCA occlusion $(A)$, which is rendered more conspicuous by delayed vessel opacification in the second and third phases (arrows in $B$ and $C$ ). length surpasses $8 \mathrm{~mm} .{ }^{15}$ The clot length is used to select the size of the stent retriever device at thrombectomy.

sCTA may overestimate the length of occlusion because of nonopacification of the vessel distal to the thrombus. Loss of the normal pressure gradient may also result in collapse of the vessel wall distal to the thrombus and may overestimate the length of occlusion (Figs 3 and 4). Clot length has been found to be subjectively easier to measure by mCTA than by catheter angiography even though mCTA is just as reliable. ${ }^{16}$

\section{Assessment of Collaterals}

There is often marked variability in the extent of neural damage and its clinical manifestation in patients with anterior circulation AIS, especially during the first few hours after onset. ${ }^{17}$ Studies have suggested that this variability is in part related to the quantity and quality of collateral circulation. ${ }^{18}$ In a 1989 study of 80 patients with AIS, Bozzao et $\mathrm{al}^{19}$ showed that more extensive collaterals assessed with conventional angiography correlated with smaller final infarct size and better prognosis. More robust collaterals have been linked to lower risk of hemorrhage after endovascular therapy. ${ }^{20}$ In patients with AIS who do not receive reperfusion treatment, good collaterals have been strongly correlated with better clinical outcomes. ${ }^{21}$ Secondary analysis of CTA collateral status from the MR CLEAN randomized clinical trial showed a positive correlation between collateral score and the degree of endovascular therapy benefit. Conversely, patients with poorer collaterals were less likely to benefit. ${ }^{5}$ Such studies suggest that collateral status may be a useful factor in the management of some patients with AIS, as proposed by the recent 2019 American Heart Association AIS guidelines. ${ }^{5}$

Interest in a time-resolved CTA technique was in part initially motivated by investigators seeking a better tool for collateral status assessment. Because collateral flow to penumbral tissue tends to disperse over time, collateral status is often better assessed using information from multiple time points. Delayed filling of pial collaterals may be underestimated by a single phase. Patients with moderate or good collaterals on mCTA can be erroneously labeled as having poor collaterals
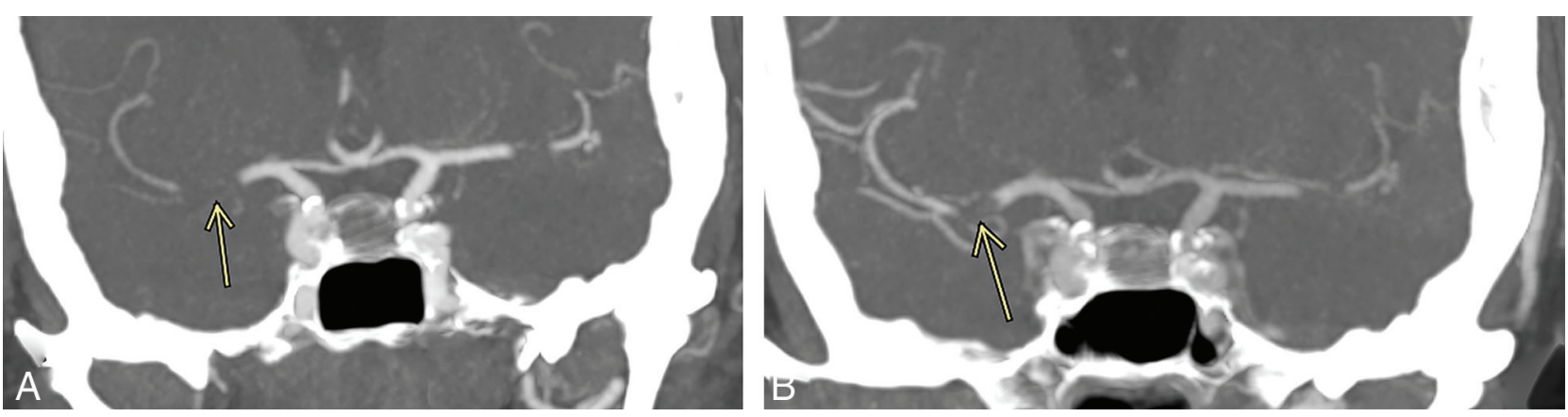

FIG 3. Coronal mCTA MIP images from the arterial phase $(A)$ and late venous phase $(B)$ show a right MCA Ml segment occlusion. The $M 1$ segment thrombus length is estimated more accurately during the venous phase ( $7 \mathrm{~mm}$ in $B$, arrow) than in the arterial phase (11 $\mathrm{mm}$ in $A$, arrow). 

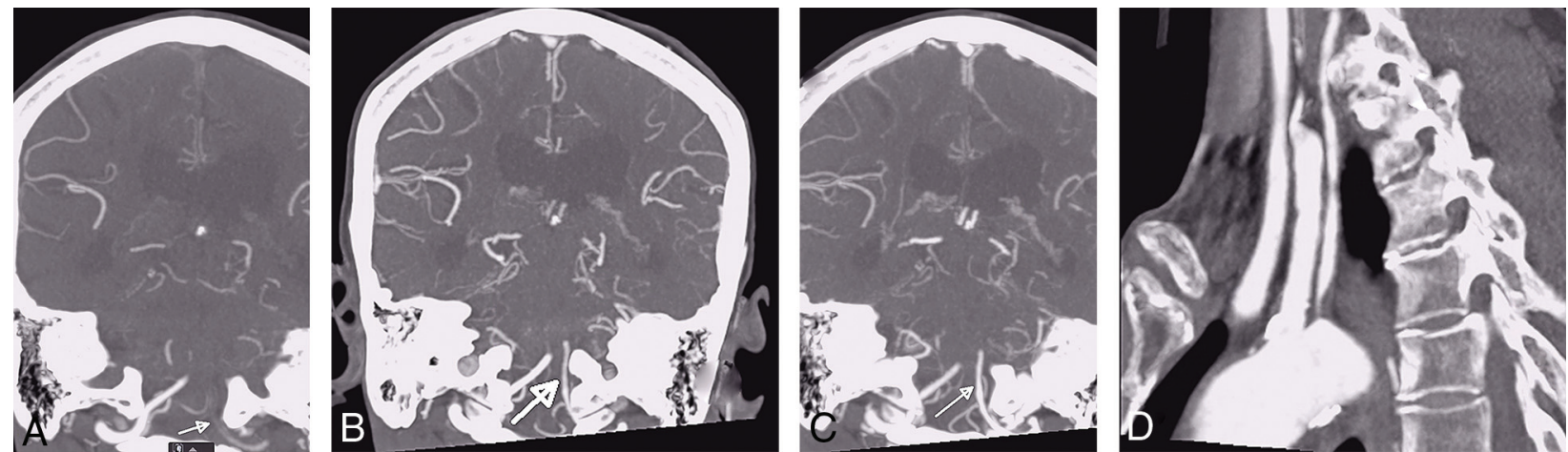

FIG 4. Pseudoocclusion of the left vertebral artery. The left vertebral artery appears occluded at its intracranial V4 segment during the arterial phase of mCTA (arrow in A). This is related to severe stenosis at its origin (D). The previously unopacified intracranial V4 segment is shown to enhance in the delayed phases of mCTA (arrows in B and C). If only single-phase CTA had been used (analogous to the arterial phase of the mCTA, A), a V4 segment occlusion would have been diagnosed.
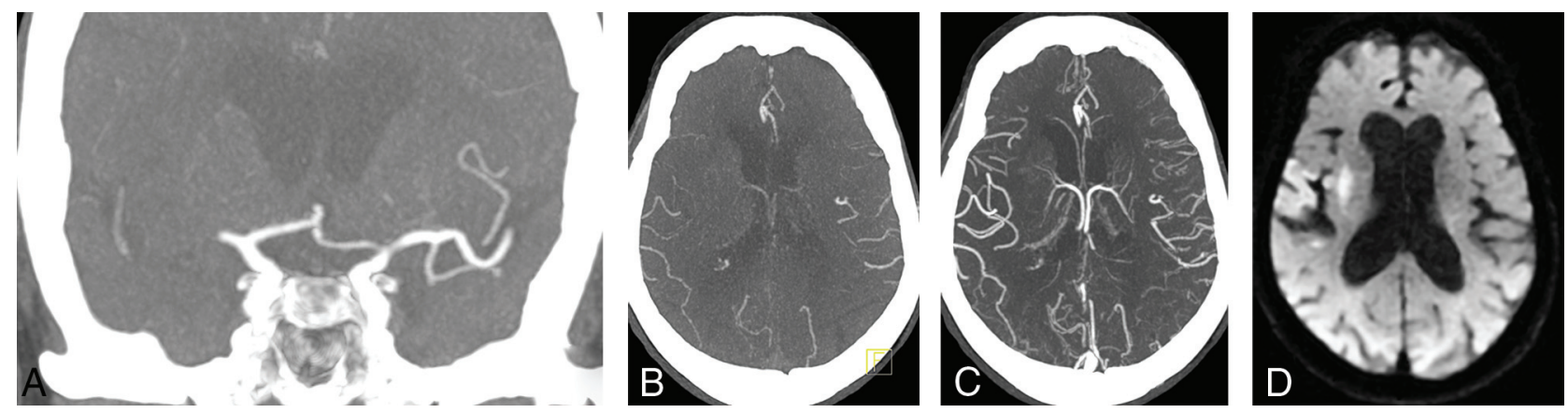

FIG 5. Coronal arterial phase mCTA MIP image demonstrates right M1 segment occlusion (A). Axial arterial phase mCTA MIP image (B) shows filling of at most $50 \%$ of distal branches. Axial delayed phase MCTA MIP image (C) shows filling of most distal branches. The patient underwent mechanical thrombectomy with restoration of $\mathrm{TICl} 2 \mathrm{c}$ flow. Follow-up diffusion-weighted MR image shows a small infarct core. If only sCTA had been used in this case (analogous to the arterial phase of the mCTA, B), the collateral status would have been classified as "poor to moderate distal collaterals" instead of "good collaterals."
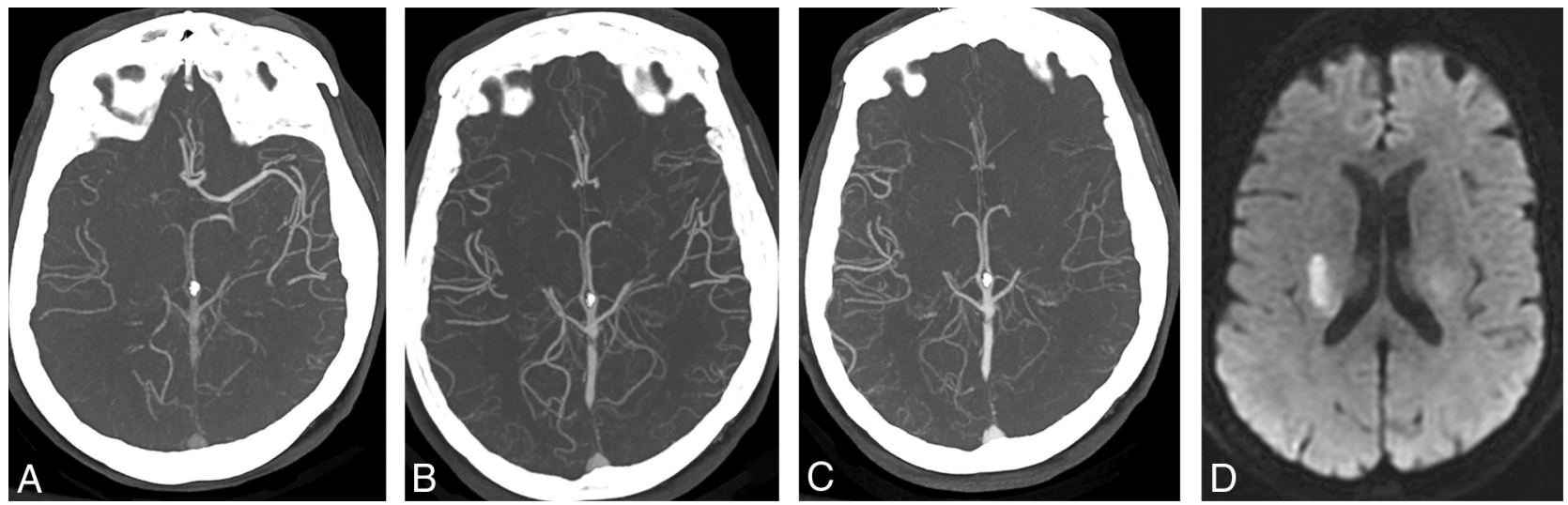

FIG 6. Right $M 1$ segment occlusion. MCTA axial MIP images from the arterial phase $(A)$, early venous phase $(B)$, and late venous phase $(C)$ show progressive collateral filling of distal MCA branches. The patient underwent endovascular recanalization with restoration of TICI 3 flow. Followup diffusion-weighted MR image shows a small infarct core.

at $\mathrm{SCTA}^{7}$ (Figs 5-7). Mis-timing of acquisition with sCTA is common, given the older age and variable cardiac status typical of the AIS population. In a study by Casault et al, ${ }^{22}$ nearly $12 \%$ of sCTA images were acquired either too early or too late, which may have resulted in mislabeling of collateral status. Pial collateral supply between PCA and MCA territories may be slower than that 
between ACA and MCA territories. ${ }^{22}$ This may contribute to limited assessment of collateral status on sCTA.

Several other techniques, including MR-based techniques and processing of CT perfusion data to create dynamic CTA, have been used for assessment of collateral circulation. Although promising, these techniques have yet to reach widespread availability. ${ }^{23}$

\section{Ischemic Core}

Rapid and accurate assessment of early infarct core and salvageable tissue is important in AIS management. Endovascular therapy has generally been considered to carry a higher risk of hemorrhage when the infarct core volume is greater than 70 to $100 \mathrm{~mL} .{ }^{24,25} \mathrm{DWI}$ is widely considered the criterion standard for core infarct assessment. However, MR imaging is not always practical in the acute stroke setting.

Perfusion studies have widely been used to estimate infarct core and penumbra volumes. Perfusion studies are typically
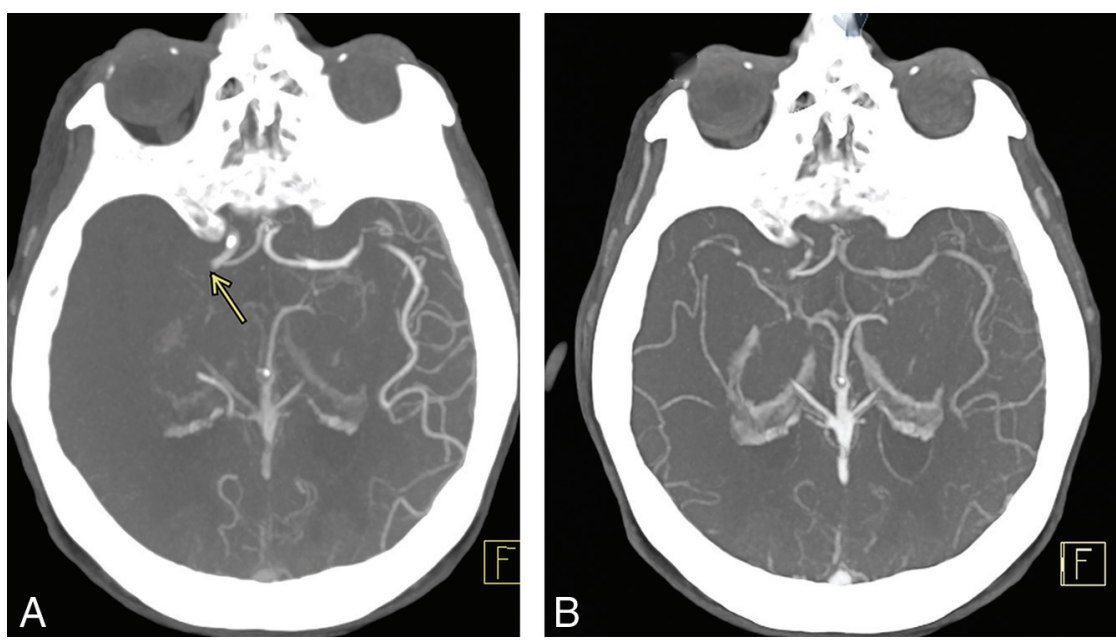

FIG 7. Multiphase CTA arterial phase axial MIP image shows occlusion of the right Ml segment (A, arrow) with no right MCA distal branches. The arterial phase of MCTA is analogous to SCTA and would have been classified as "poor collaterals" in this patient, if considered without the benefit of delayed-phase CTA images. MCTA delayed phase axial MIP in the same patient (B) shows filling of about $50 \%$ of right MCA distal branches, resulting in a more accurate assessment of collateral status.
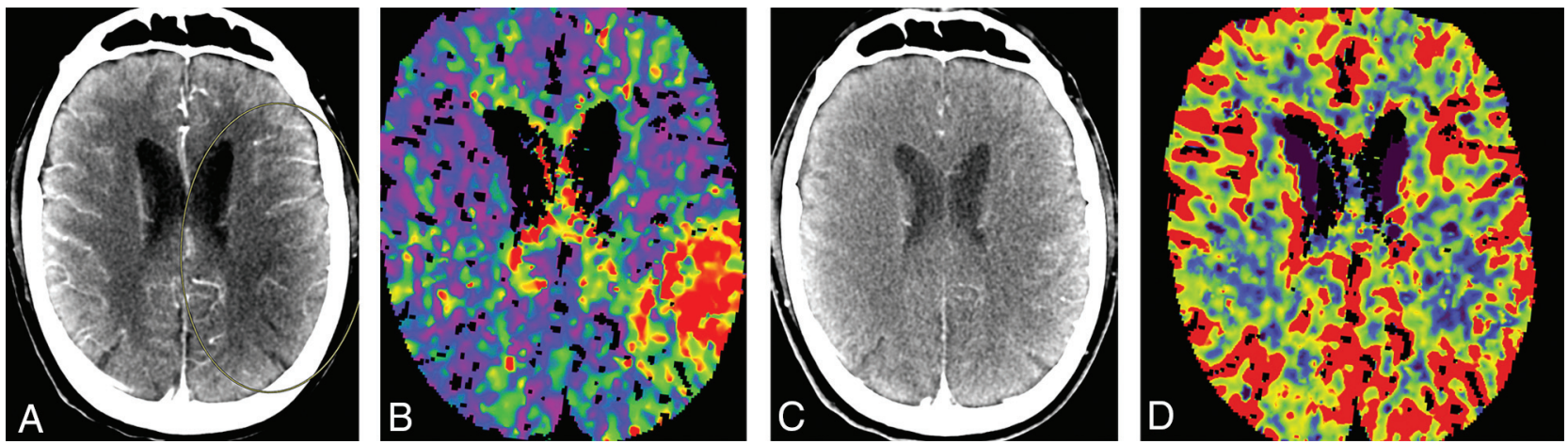

FIG 8. Correlation between $\mathrm{MCTA}$ and $C T$ perfusion imaging in a patient with left MCA M2 segment occlusion. A region of hypoattenuation in an arterial phase mCTA source image ( $A$, circled area) closely correlates with a region of delayed Tmax in a CT perfusion image ( $B$ ), suggestive of tissue at risk. Absence of hypoattenuation in a delayed venous phase mCTA source image $(C)$ is matched by preservation of cerebral blood volume in a CT perfusion image $(D)$, suggestive of no infarct core. The patient underwent endovascular intervention with restoration of TICI $2 \mathrm{~b}$ antegrade flow. Subsequent diffusion-weighted MR imaging showed only small foci of acute ischemia in the frontoparietal region (not shown). 
mCTA to be superior to sCTA and CTP in determining clinical outcome. In contrast, secondary analysis of data from the DEFUSE 3 randomized clinical trial by de Havenon et $\mathrm{al}^{28}$ showed no correlation between good collaterals assessed by CTA and improved clinical outcome. Among the limitations cited by de Havenon et $\mathrm{al}^{28}$ were the possibility of continued collateral status evolution after imaging assessment and the use of sCTA. Zhang et $\mathrm{al}^{29}$ found that good collaterals are better predictors of favorable outcome in the cardioembolism stroke subtype than in the large artery atherosclerosis stroke subtype.

\section{Radiation Dose}

The mean estimated effective dose of mCTA is typically only $20 \%$ above that of sCTA compared with 70\% added dose for CT perfusion. ${ }^{7,30}$ The added radiation dose to the eye is typically 4 times higher with CT perfusion than with mCTA. ${ }^{7}$

\section{CT Perfusion and mCTA}

The DAWN ${ }^{2}$ and DEFUSE $3^{3}$ randomized clinical trials have shown benefit from endovascular therapy in the extended time window (6-24 hours), provided that the infarct core was small and there was a large amount of tissue at risk. The 2019 American Heart Association AIS guidelines recommend obtaining CTP or DWI with or without MR perfusion in the extended time window after anterior circulation large-vessel occlusion. ${ }^{1}$ However, the AHA guidelines also suggest that collateral assessment may be useful in selecting some patients for mechanical thrombectomy, citing the use of mCTA collateral assessment for patient selection in the ESCAPE randomized clinical trial. ${ }^{4}$ Secondary analysis from the MR CLEAN trial has also supported the utility of collateral status. ${ }^{5}$ These initial studies have prompted increased use of mCTA in recent randomized clinical trials. Definitive recommendations on the role of mCTA in AIS management will await the completion of such trials.

The improved vessel occlusion detection rate, higher interrater reliability, improved measurement of thrombus length, and relatively low costs of mCTA support its use in lieu of sCTA as the standard initial vessel imaging test in the AIS setting. mCTA may be more resistant to patient motion, poor hemodynamics, and other technical factors compared with sCTA or CTP.

\section{Limitations}

As with any other test, mCTA should be interpreted in conjunction with clinical information and other available imaging, including NCCT. Flow-limiting stenosis in proximal neck vessels can potentially delay opacification of pial vessels in both ischemic and nonischemic tissue and thus lead to suboptimal assessment of collateral status. Poor hemodynamics can result in poor pial vessel opacification and thereby limit the benefits of temporal resolution at vessel imaging.

mCTA is of limited value in posterior circulation AIS, except in cases involving the posterior cerebral artery. ${ }^{7}$ This is likely related to the highly variable vascular anatomy of the posterior circulation and thus its poorly understood collateral supply.

\section{CONCLUSIONS}

In summary, mCTA offers several significant advantages over the traditional sCTA technique in AIS assessment, including improved detection of vessel occlusion, improved collateral status assessment, improved tolerance of suboptimal patient factors, and higher interrater reliability. ${ }^{26}$ These benefits are gleaned at little additional cost in terms of time, risk to the patient, and capital expense. Although future study results will be needed to ascertain the role of mCTA in relation to perfusion techniques and DWI in the extended time window, existing data already suggest that there are important benefits to using mCTA in lieu of sCTA in the initial vessel assessment of patients with acute stroke.

\section{REFERENCES}

1. Powers WJ, Rabinstein AA, Ackerson T, et al. Guidelines for the Early Management of Patients With Acute Ischemic Stroke: 2019 Update to the 2018 Guidelines for the Early Management of Acute Ischemic Stroke: A Guideline for Healthcare Professionals From the American Heart Association/American Stroke Association. Stroke 2019;50:e344418 CrossRef Medline

2. Nogueira RG, Jadhav AP, Haussen DC, et al. Thrombectomy 6 to 24 hours after stroke with a mismatch between deficit and infarct. $N$ Engl J Med 2018;378:11-21 CrossRef Medline

3. Albers GW, Marks MP, Kemp S, et al. Thrombectomy for stroke at 6 to 16 hours with selection by perfusion imaging. $N$ Engl J Med 2018;378:708-18 CrossRef Medline

4. Goyal M, Demchuk AM, Menon BK, et al. Randomized assessment of rapid endovascular treatment of ischemic stroke. $N$ Engl J Med 2015;372:1019-30 CrossRef Medline

5. Berkhemer OA, Jansen IGH, Beumer D, et al. Collateral status on baseline computed tomographic angiography and intra-arterial treatment effect in patients with proximal anterior circulation stroke. Stroke 2016;47:768-76 CrossRef Medline

6. Yu AYX, Zerna C, Assis Z, et al. Multiphase CT angiography increases detection of anterior circulation intracranial occlusion. Neurology 2016;87:609-16 CrossRef Medline

7. Menon BK, d'Esterre CD, Qazi EM, et al. Multiphase CT angiography: a new tool for the imaging triage of patients with acute ischemic stroke. Radiology 2015;275:510-20 CrossRef Medline

8. Ospel JM, Volny O, Qiu W, et al. Displaying multiphase CT angiography using a time-variant color map: practical considerations and potential applications in patients with acute stroke. AJNR Am J Neuroradiol 2020;41:200-05 CrossRef Medline

9. Volny O, Cimflova P, Kadlecova P, et al. Single-phase versus multiphase CT angiography in middle cerebral artery clot detectionbenefits for less experienced radiologists and neurologists. J Stroke Cerebrovasc Dis 2017;26:19-24 CrossRef Medline

10. Byrne D, Sugrue G, Stanley E, et al. Improved detection of anterior circulation occlusions: the "delayed vessel sign" on multiphase CT angiography. AJNR Am J Neuroradiol 2017;38:1911-16 CrossRef Medline

11. Tomsick TA, Carrozzella J, Foster L, et al. Endovascular therapy of M2 occlusion in IMS III: role of M2 segment definition and location on clinical and revascularization outcomes. AJNR Am J Neuroradiol 2017;38:84-89 CrossRef Medline

12. Sarraj A, Sangha N, Hussain MS, et al. Endovascular therapy for acute ischemic stroke with occlusion of the middle cerebral artery M2 segment. JAMA Neurol 2016;73:1291-96 CrossRef Medline

13. Coutinho JM, Liebeskind DS, Slater L-A, et al. Mechanical thrombectomy for isolated M2 occlusions: a post hoc analysis of the STAR, SWIFT, and SWIFT PRIME studies. AJNR Am J Neuroradiol 2016;37:667-72 CrossRef Medline

14. Puetz V, Dzialowski I, Hill MD, et al. Intracranial thrombus extent predicts clinical outcome, final infarct size and hemorrhagic 
transformation in ischemic stroke: the clot burden score. Int $J$ Stroke 2008;3:230-36 CrossRef Medline

15. Riedel CH, Zimmermann P, Jensen-Kondering U, et al. The importance of size: successful recanalization by intravenous thrombolysis in acute anterior stroke depends on thrombus length. Stroke 2011;42:1775-77 CrossRef Medline

16. Polito V, La Piana R, Del Pilar Cortes M, et al. Assessment of clot length with multiphase CT angiography in patients with acute ischemic stroke. Neuroradiol J 2017;30:593-99 CrossRef Medline

17. Yeo LLL, Paliwal P, Teoh HL, et al. Assessment of intracranial collaterals on CT angiography in anterior circulation acute ischemic stroke. AJNR Am J Neuroradiol 2015;36:289-94 CrossRef Medline

18. Liebeskind DS, Kim D, Starkman S, et al. Collateral failure? Late mechanical thrombectomy after failed intravenous thrombolysis. $J$ Neuroimaging 2010;20:78-82 CrossRef Medline

19. Bozzao L, Fantozzi LM, Bastianello S, et al. Early collateral blood supply and late parenchymal brain damage in patients with middle cerebral artery occlusion. Stroke 1989;20:735-40 CrossRef Medline

20. Bang OY, Saver JL, Kim SJ, et al. Collateral flow averts hemorrhagic transformation after endovascular therapy for acute ischemic stroke. Stroke 2011;42:2235-39 CrossRef Medline

21. Lima FO, Furie KL, Silva GS, et al. The pattern of leptomeningeal collaterals on CT angiography is a strong predictor of long-term functional outcome in stroke patients with large vessel intracranial occlusion. Stroke 2010;41:2316-22 CrossRef Medline
22. Casault C, Al Sultan AS, Trivedi A, et al. Collateral scoring on CT angiogram must evaluate phase and regional pattern. Can J Neurol Sci J Can Sci 2017;44:503-07 CrossRef Medline

23. Bang OY, Goyal M, Liebeskind DS. Collateral circulation in ischemic stroke: assessment tools and therapeutic strategies. Stroke 2015;46:3302-09 CrossRef Medline

24. Lansberg MG, Thijs VN, Bammer R, et al. Risk factors of symptomatic intracerebral hemorrhage after tPA therapy for acute stroke. Stroke 2007;38:2275-78 CrossRef Medline

25. Mlynash M, Lansberg MG, De Silva DA, et al. Refining the definition of the malignant profile: insights from the DEFUSE-EPITHET pooled data set. Stroke 2011;42:1270-75 CrossRef Medline

26. Reid M, Famuyide AO, Forkert ND, et al. Accuracy and reliability of multiphase CTA perfusion for identifying ischemic core. Clin Neuroradiol 2019;29:543-52 CrossRef Medline

27. Santos EMM, d'Esterre CD, Treurniet KM, et al. Added value of multiphase CTA imaging for thrombus perviousness assessment. Neuroradiology 2018;60:71-79 CrossRef Medline

28. de Havenon A, Mlynash M, Kim-Tenser MA, et al. Results from DEFUSE 3: good collaterals are associated with reduced ischemic core growth but not neurologic outcome. Stroke 2019;50:632-38 CrossRef Medline

29. Zhang X, Zhang M, Ding W, et al. Distinct predictive role of collateral status on clinical outcome in variant stroke subtypes of acute large arterial occlusion. Eur J Neurol 2018;25:293-300 CrossRef Medline

30. Yang C-Y, Chen Y-F, Lee C-W, et al. Multiphase CT angiography versus single-phase CT angiography: comparison of image quality and radiation dose. AJNR Am J Neuroradiol 2008;29:1288-95 CrossRef Medline 